\title{
Successful Tavi in Patients with Previous Mitral Valve Replacement
}

\author{
Matjaž Bunc ${ }^{1,2,3^{*}}$, Jana Ambrozic ${ }^{1}$, Špela Mušič ${ }^{4}$, Nikola Lakič $\breve{c o s}^{3,4}$ P. Berden ${ }^{5}$, Rok Zbačnik ${ }^{5}$, \\ Darko Zorman ${ }^{1,3}$
}

${ }^{1}$ Department of Cardiology, University Medical Centre Ljubljana, Ljubljana, Slovenia; ${ }^{2}$ Institute for Pathophysiology, School of Medicine Ljubljana, Ljubljana, Slovenia; ${ }^{3}$ Cerklje na Gorenjskem, Trg Davorina Jenka, Cerklje na Gorenjskem; ${ }^{4}$ Department of Cardiovascular Surgery, University Medical Centre Ljubljana, Ljubljana, Slovenia; ${ }^{5}$ Clinical Institute of Radiology, University Medical Centre Ljubljana, Ljubljana, Slovenia.

Email: *mbuncek@yahoo.com, jana.ambrozic@gmail.com

Received September $6^{\text {th }}, 2012$; revised October $30^{\text {th }}, 2012$; accepted November $24^{\text {th }}, 2012$

\begin{abstract}
Background: Transcatheter aortic valve implantation (TAVI) is a new method for patients with severe aortic stenosis at high surgical risk, such as previous cardiac surgery. The presence of mechanical mitral prosthesis might complicate TAVI because of possible interference between both prostheses. Some reports have already demonstrated the feasibility of TAVI in such patients. Case Reports: We report 2 patients with severe symptomatic aortic stenosis who had prior mitral valve replacement that successfully underwent TAVI with Edwards Sapien and CoreValve aortic prosthesis, respectively. Computed tomographic angiography (CTA) was useful for the assessment of the distance between both aortic and mitral prosthesis annuli. Transesophageal echocardiography was crucial for precise positioning of TAV. There were no special technical tips besides precise positioning of the valve prosthesis. In case of Core Valve the goal was the positioning at "zero point" and in case of Edwards Sapien valve a "half-on-half" position according to natural aortic valve. We observed no deformation or dysfunction of aortic and mitral prosthesis in any of the patients. Balloon valvuloplasty prior to implantation helps to observe the mutual effect of the new aortic valve and pre-existent mitral prosthesis. Conclusions: We conclude that TAVI can be safely and successfully performed in patients with mechanical mitral prosthesis carefully considering the altered anatomical conditions. Skillfulness and TAVI experience of the operators should not be neglected.
\end{abstract}

Keywords: TAVI; Mitral Valve Replacement; Aortic Prosthesis; Mitral Prosthesis

\section{Background}

Transcatheter aortic valve implantation (TAVI) is a relatively new option for the treatment of severe symptomatic aortic stenosis in high-risk patients not being considered for surgery because of significant comorbidities [1]. Using the percutaneous approach the procedural risk can be considerably reduced also in a cohort of patients who have had previous cardiac surgery. On the other hand, TAVI can be complicated in patients with previous mitral valve replacement due to possible interference between the mechanical mitral and percutaneous aortic valve prosthesis. Therefore the presence of mitral valve prosthesis is considered a contraindication for TAVI in the currently ongoing Placement of AoRTic TraNscathetER Valve (PARTNER) Trial, which compares TAVI, surgical aortic valve replacement and medical therapy [2]. In spite of this, some case reports have

*Corresponding author. emerged in the last years showing the feasibility of TAVI via transfemoral as well as transapical approach with both available percutaneous aortic prostheses (Edwards Sapien, Edwards Lifesciences, Inc., Irvine, California and CorveValve, Inc., Irvine, California) in patients with artificial mitral valve [3-8].

TAVI in a patient with pre-existent mechanical mitral valve should be considered with caution due to anatomical close proximity of the aortic and mitral annuli [3-6]. There are several concerns that should be kept in mind: 1) the risk of the aortic valve under-expansion in relation to the noncompliant mechanical mitral prosthesis, also due to significant reduction of the mitro-aortic space limiting the accommodation especially of the balloon-expandable valve; 2) the risk of embolization of the device; and 3) the risk of post-procedural dysfunction of the mitral prosthesis due to its damage during percutaneous manipulation or interference between the prosthesis and distal edge of the aortic valve. The authors reported the impor- 
tance of various methods for guidance: balloon aortic valvuloplasty of a similar balloon size to the valve stent for assessing the expansion and stability of the balloon and no interference with the mitral prosthesis, fluoroscopy and transesophageal echocardiography (TEE) to observe the correct position of the device in relation to the mitral prosthesis and cardiac computed tomographic angiography (CTA) for the assessment of the distance between both annuli and amount of excursion available for the stented valve [3-8].

Recently published cases showed that considering aforementioned conditions TAVI is a feasible option in such patients [3-8]. In this paper we demonstrate our experience with TAVI in 2 patients after previous mitral valve replacement.

In University Medical Center Ljubljana, Slovenia, TAVR program was started in October 2009. We have experiences with implantation of both CoreValve (Medtronic, USA) and Edwards-Sapien (Edwards, USA) valves. The TAVR program was approved by Slovenian Ethic Committee, Scientific Board of University Medical Center of Ljubljana and Ministry of Health of Slovenia.

\section{Case Reports}

\subsection{Patient 1}

A 77-year-old woman (weight $68 \mathrm{~kg}$, height $168 \mathrm{~cm}$, RR: $155 / 85 \mathrm{mmHg}$ ) presented with progressive shortness of breath and recurrent syncope. Six years ago she underwent a mitral valve repair with a ring annuloplasty due to mitral valve endocarditis. One year after surgery a St. Jude bileaflet mechanical prosthesis (St. Jude Medical, Inc., St. Paul, Minnesota) no. 29 was implanted for severe mitral regurgitation. On admission echocardiogramphy showed severe aortic stenosis with an aortic valve area (AVA) of $0.6 \mathrm{~cm}^{2}$ and a mean gradient of $43 \mathrm{mmHg}$. The operative mortality risk was $16.1 \%$ according to the logistic EuroScore. The patient refused the third cardiac surgery. Since TAVI was not available in our institution we decided to perform balloon aortic valvuloplasty (BAV) in attempt to relief her symptoms. After transient improvement six months later she was readmitted. At that time TAVI was accessible in our institution. After evaluation she was scheduled for the no. 23 Edwards Sapien prosthesis.

Patients data: weight 68 kg, RR: 155/85 mmHg.

\subsection{Patient 2}

An 84-year-old man (weight $73 \mathrm{~kg}$, height $171 \mathrm{~cm}$, RR: $170 / 95 \mathrm{mmHg}$ ) presented with a shortness of breath and angina pectoris. In 2003 he had endoscopic mitral valve replacement with a St. Jude bileaflet mechanical prosthesis no. 31 for severe mitral regurgitation due to flail leaf- let. Echocardiography revealed severe aortic stenosis with an AVA of $0.7 \mathrm{~cm}^{2}$ and a mean gradient of 38 $\mathrm{mmHg}$, depressed left ventricular ejection fraction of $35 \%$ and severe pulmonary hypertension. Coronary angiography showed no coronary stenosis. The logistic Euroscore was $41.8 \%$. After the patient evaluation we decided to perform TAVI with the CoreValve prosthesis no. 26.

The patients underwent TAVI in our cardiac catheterization laboratory in local anesthesia and mild sedation by a team of cardiologists, cardiac surgeon and anesthesiologist. BAV showed a good expansion and stability of the balloon. Via femoral approach the Edwards Sapien prosthesis in the first patient and the CoreValve prosthesis in the second one were introduced and after careful positioning under fluoroscopy and TEE guidance the valves were implanted. Transesophageal echocardiography was crucial for precise positioning of TAV. There were no special technical tips besides precise positioning of the valve prosthesis. In case of Core Valve the goal was the positioning at "zero point" and in case of Edwards valve a "half-on-half" position according to natural aortic valve.

During CoreValve expansion we used rapid pacing of left ventricle at rate $110 / \mathrm{min}$. Under diascopy and temporary aortic angiography we checking opening of the valve and taking care about the optimal position of the valve. The crucial point was not to start to deep in outflow tract of left ventricle. In this particular case we stared just one diamond below the zero point and keep tension on the valve. After complete distal part opening we leave valve a bit to orient and than we pull the valve up and keep it at zero point until finishing the expansion. The optimal positioning of the valve was checking also with TEE probe. We stop the rapid pacing after opening the CoreValve over the artificial leaflets position.

In case of Edwards Sapien valve the prosthesis was set in a predicted position; half and half according to natural aortic valve. We checked the position and relation to artificial mitral valve by TEE. With rapid pacing up to 190/min we checked the valve position stability. During rapid pacing we delivered the valve in two phase balloon inflation: first slow, with option of repositioning and second fast inflation of the rest $20 \%$ of the inflation balloon.

Subsequently, fluoroscopy and TEE showed a good position of the aortic prostheses with mild paravalvular leaks and no interference between the both prostheses (Figures 1 and 2). We observed no deformation or dysfunction of aortic and mitral prosthesis in any of the patients during 5 days hospital stay after TAVI. Three-dimensional TEE and CTA images, shown in Figures 1 and $\mathbf{2}$, demonstrate the close proximity of both prostheses. There were no complications related to the procedure. 


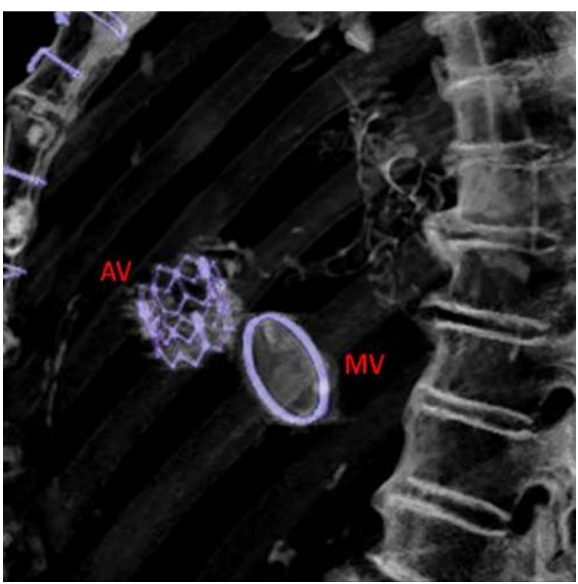

(a)

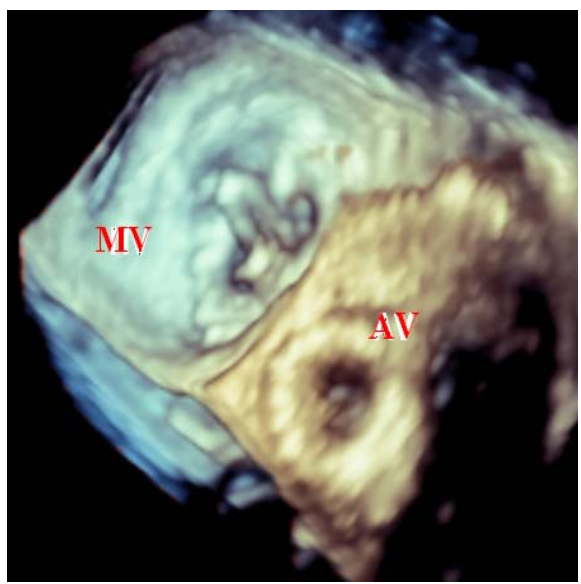

(b)

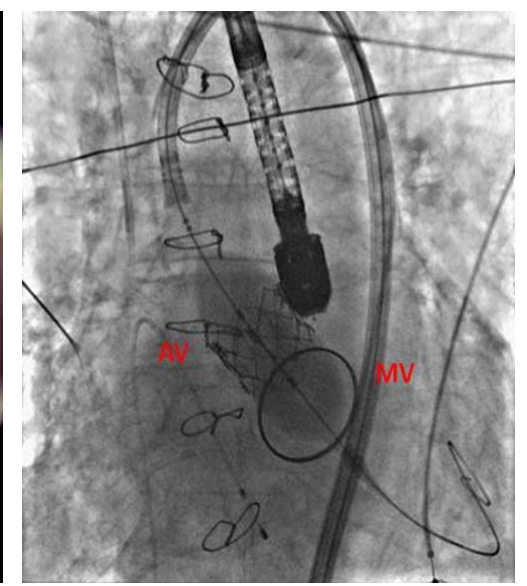

(c)

Figure 1. Patient 1: (a) Fluoroscopic image of the implantation of the balloon-expandable Edwards Sapien aortic valve showing a close proximity of both prostheses with no interference between them; (b) Three-dimensional TEE image of the mitral mechanical and Edwards Sapien aortic prosthesis; (c) Three-dimensional 3D CTA image 6 months after TAVI: the position of the Edwards Sapien valve and its relation to mechanical mitral prosthesis is nicely seen. $\mathrm{AV}=$ aortic prosthesis; $\mathrm{MV}=$ mitral prosthesis.

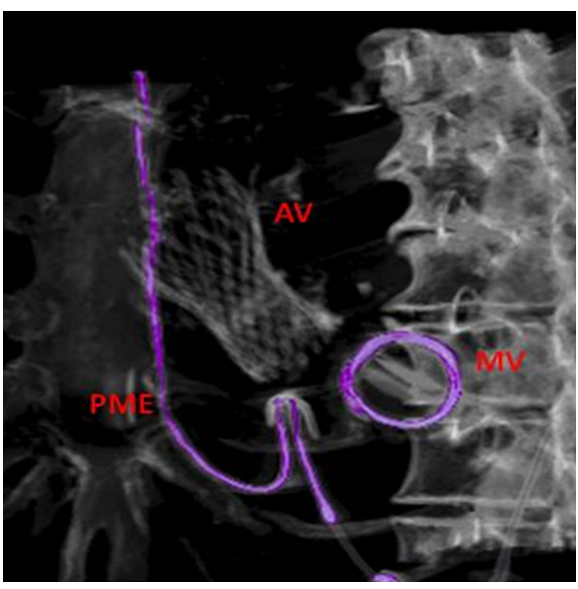

(a)

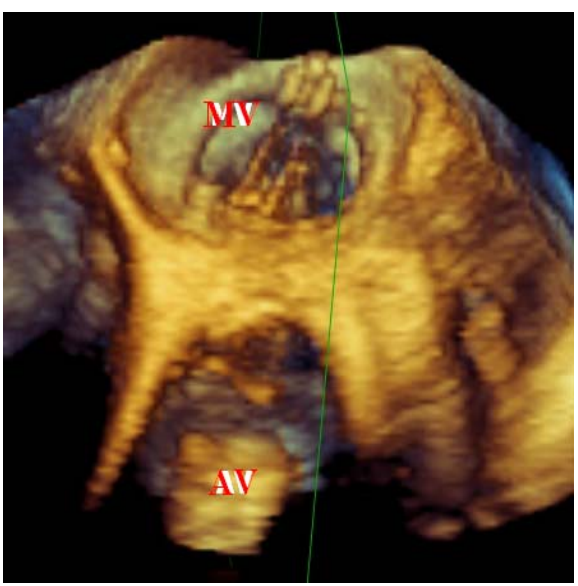

(b)

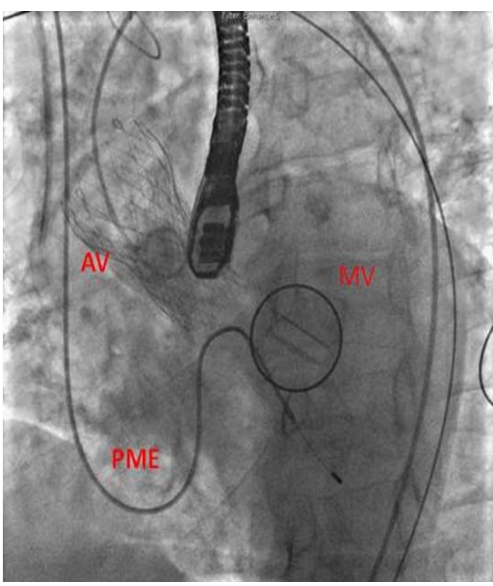

(c)

Figure 2. Patient 2: (a) Fluoroscopy after TAVI: successful implantation of the CoreValve aortic prosthesis with no evidence of interference of the aortic with the mitral bileaflet prosthesis; (b) Three-dimensional TEE image of the mitral mechanical and CoreValve aortic prosthesis; (c) Three-dimensional CTA image after TAVI showing the CoreValve prosthesis in aortic position and mechanical bileaflet prosthesis in mitral position. $\mathrm{AV}=$ aortic prosthesis; $\mathrm{MV}=$ mitral prosthesis; $\mathrm{PME}=$ pacemaker electrode in the right chambers.

During the mean follow-up of one year we still observe symptomatic improvement in both patients and a good function of both valve prostheses.

\section{Discussion and Conclusions}

The present cases demonstrate that TAVI can be successfully performed in patients with previous mitral valve surgery. There have already been reports published showing the feasibility of this treatment option in such situations, even though the mitral prosthesis was initially considered an exclusion criteria for TAVI [3-8]. Special caution is needed to achieve a good position and proper expansion of the aortic valve without interference and damage of the existent mitral prosthesis. In order to assess properly the altered anatomical conditions related to reduced aorto-mitral space and loss of fibrous tissue limiting the valve stent expansion some authors suggested CTA pre-operatively [6], during procedure, however, three-dimensional TEE allows a more accurate assessment of the anatomy. Balloon valvuloplasty as a part of the TAVI procedure for pre-dilation of the native aortic valve is a very useful method. In this setting BAV allows us to assess the ability of the aortic valve expansion and its possible interaction with mitral valve function $[3,6]$.

In our center we successfully performed over 290 
BAV procedures in high risk patients with severe symptomatic aortic stenosis as a palliative measure. The experience with BAV, which is usually part of the preparation for TAVI, helped us to start the TAVI program with more confidence.

In case of TAVI in patients after mitral valve replacement the operator skills and TAVI experiences are important. We performed the presented cases as our $15^{\text {th }}$ CoreValve procedure and $12^{\text {th }}$ Edwards-Sapien case.

Althought there are no special technical tips besides precise positioning of the valve prosthesis, such a procedure it should not be performed among first ten TAVI cases, in a certain center.

\section{REFERENCES}

[1] J. G. Webb, L. Altwegg, R. H. Boone, et al., "Transcatheter Aortic Valve Implantation: Impact on Clinical and Valve-Related Outcomes," Circulation, Vol. 119, 2009, pp. 3009-3016. doi:10.1161/CIRCULATIONAHA.108.837807

[2] “Placement of AoRTic TranscathetER Valve (PARTNER) trial,” NCT00530894, 2012.

http://clinicaltrials.gov/ct2/show/NCT00530894

[3] J. Rodés-Cabau, E. Dumont, S. Miró, et al., “Apical Aortic Valve Implantation in a Patient with a Mechanical Valve Prosthesis in Mitral Position,” Circulation: Cardio- vascular Interventions, Vol. 1, 2008, p. 233. doi:10.1161/CIRCINTERVENTIONS.108.813592

[4] N. Dumonteil, B. Marcheix, P. Berthoumieu, et al., “Transfemoral Aortic Valve Implantation with Pre-Existent Mechanical Mitral Prosthesis: Evidence of Feasibility,” JACC: Cardiovascular Interventions, Vol. 2, No. 9, 2009, pp. 897-898. doi:10.1016/j.jcin.2009.05.023

[5] G. Bruschi, F. De Marco, J. Oreglia, et al., "Percutaneous Implantation of CoreValve Aortic Prostheses in Patients with a Mechanical Mitral Valve," Annals of Thoracic Surgery, Vol. 88, No. 5, 2009, pp. 50-52. doi:10.1016/j.athoracsur.2009.07.028

[6] V. T. Chao, P. T. Chiam and S. Y. Tan, "Transcatheter Aortic Valve Implantation with Preexisting Mechanical Mitral Prosthesis-Use of CT Angiography," Journal of Invasive Cardiology, Vol. 22, No. 7, 2010, pp. 339-340.

[7] M. Scherner, J. T. Strauch, P. L. Haldenwang, F. Baer and T. Wahlers, "Successful Transapical Aortic Valve Replacement in a Patient with Previous Mechanical Mitral Valve Replacement,” Annals of Thoracic Surgery, Vol. 88, No. 5, 2009, pp. 1662-1663. doi:10.1016/j.athoracsur.2009.04.078

[8] C. J. Beller, R. Bekeredjian, U. Krumsdorf, et al., “Transcatheter Aortic Valve Implantation after Previous Mechanical Mitral Valve Replacement: Expanding Indications?” Heart Surgery Forum, Vol. 14, No. 3, 2011, pp. 166-170. doi:10.1532/HSF98.20101148 